Published in final edited form as:

Transplantation. 2017 June ; 101(6): 1381-1386. doi:10.1097/TP.0000000000001262.

\title{
Outcomes of Solid Organ Transplants After Simultaneous Solid Organ and Vascularized Composite Allograft Procurements: A Nationwide Analysis
}

\author{
Mario A. Aycart, MD" ${ }^{\# 1}$, Muayyad Alhefzi, MD\#1, Gaurav Sharma, MD², Nicco Krezdorn, MD1, \\ Ericka M. Bueno, PhD ${ }^{1}$, Simon G. Talbot, MD ${ }^{1}$, Matthew J. Carty, MD ${ }^{1}$, Stefan G. Tullius, MD, \\ PhD $^{3}$, and Bohdan Pomahac, MD ${ }^{1}$ \\ ${ }^{1}$ Division of Plastic Surgery, Department of Surgery, Brigham and Women's Hospital, Harvard \\ Medical School, Boston, MA. \\ 2Department of Surgery, Brigham and Women's Hospital, Harvard Medical School, Boston, MA. \\ 3Division of Transplant Surgery, Department of Surgery, Brigham and Women's Hospital, Harvard \\ Medical School, Boston, MA. \\ \# These authors contributed equally to this work.
}

\begin{abstract}
Background.-Current knowledge of the impact of facial vascularized composite allograft (VCA) procurement on the transplantation outcomes of the concomitantly recovered solid organs is limited to isolated case reports and short-term results. Here we report on a nationwide analysis of facial allograft donor surgery experience and long-term outcomes of the concomitantly recovered solid organs and their recipients.
\end{abstract}

\begin{abstract}
Methods.-There were 10 facial VCA procurements in organ donors between December 2008 and October 2014. We identified the population of subjects who received solid organs from these 10 donors using the Scientific Registry of Transplant Recipients. We retrospectively reviewed operative characteristics, intraoperative parameters, and postoperative outcomes.
\end{abstract}

\begin{abstract}
Results.-Six of 10 donor surgeries were performed at outside institutions, all on brain-dead donors. Mean operative duration for facial VCA recovery was 6.9 hours (range, 4-13.25 hours). A total of 36 solid organs were recovered and transplanted into 35 recipients. Survival rates for kidney and liver recipients were $100 \%$ and $90 \%$ at a median follow-up of 33 and 27.5 months, respectively (range, 6-72 months). Graft survival rates for kidneys and livers were 15 of 16 (94\%) and 9 of 10 (90\%), respectively. Recipient and graft survival rates for hearts and lungs were $75 \%$ $(\mathrm{n}=4)$ and $100 \%(\mathrm{n}=3)$ at mean follow-up time of 14.75 and 16 months, respectively. A liver
\end{abstract}

\footnotetext{
Correspondence: Bohdan Pomahac, MD, Plastic Surgery Transplantation and Burn Center, Brigham and Women's Hospital, Harvard Medical School, 75 Francis Street, Boston, MA 02115 (bpomahac@ partners.org).

M.A.A. and M.A. contributed to the conception and design. M.A.A., M.A., and G.S. collected the data. M.A.A., M.A., and G.S. performed the data analysis. M.A.A., MA, G.S., N.K., E.M.B., S.G.T., and B.P. contributed to data interpretation.M.A.A, MA, and G.S. drafted the article. M.A.A, M.A, G.S., N.K., E.M.B., S.G.T,M.J.C., S.G.T., and B.P. contributed to revision and final approval of the article.

The authors declare no conflicts of interest.

Our clinical trial for Face Transplantation is registered at ClinicalTrials.govas identifier, NCT01281267.
} 
recipient died at 22 months from unknown causes and a heart recipient died of leukemia at 10 months.

Conclusions.-Facial VCA procurement does not appear to adversely affect the outcomes of transplant recipients of concomitantly recovered solid organ allografts.

Face transplantation has emerged atop the reconstructive ladder as the only true restorative therapy for the most complex facial defects. Though still considered experimental, face transplantation has proven to be life-changing with superior results over conventional methods for the most severely disfigured patients. ${ }^{1-3}$ To our knowledge, there have been approximately 37 face transplants performed world-wide. Although the logistics and technical aspects of the donor facial vascularized composite allograft (VCA) procurement have been previously detailed, ${ }^{4-10}$ study of the impact of facial VCA recovery on the outcomes of concomitantly recovered solid organ allografts has been limited. Facial VCA procurement is technically challenging and full facial procurement has, in some cases, exceeded 12 hours. ${ }^{9}$ Thus, "simultaneous-start" procurement of lifesaving organs and facial VCAs could result in prolonged ischemia time to the facial VCA. Therefore, some institutions, including our own, utilize a "face-first" protocol in hemodynamically stable, heart-beating donors. ${ }^{4}$

Concomitant multi-organ procurement including the whole face has been reported to be safe and feasible without short-term jeopardy to lifesaving organs in isolated case reports. ${ }^{8-10}$ Although these individual cases are important to the medical literature and the burgeoning field of reconstructive transplantation, experience from larger series with longer-term outcome data for transplant recipients and their respective solid organs has not been reported. We sought to critically review our institutional and national facial VCA donor surgery experience to quantify the impact on the organs procured and transplanted from facial and multi-VCA donors.

\section{MATERIALS AND METHODS}

This study was exempt from review by our institutional review board. The study population was identified using data from the Scientific Registry of Transplant Recipients (SRTR).The study period included follow-up data through September 25, 2015. The SRTR data system includes data on all donor, waitlisted candidates, and transplant recipients in the United States, as reported by the members of the Organ Procurement and Transplantation Network. This study was approved by the Health Resources and Services Administration's SRTR project officer. We reviewed data from our regional organ procurement organization - the New England Organ Bank, regarding our program's donor surgeries from April 2009 to October 2014 as well as operative and anesthetic records. We examined the peer-reviewed literature for data regarding other US centers' experience with additional information obtained from Lifebanc of Ohio and The Living Legacy Foundation of Maryland. We collected relevant donor characteristics, such as age, sex, body mass index calculated as weight $(\mathrm{kg}) /$ height $\left(\mathrm{m}^{2}\right)$, in-hospital duration of stay (ie, days) before procurement, history of hypertension or diabetes, anti-hepatitis $\mathrm{C}$ virus re-activity, and cause of death. Through the SRTR, we also collected terminal laboratory results, such as serum sodium $(\mathrm{mEq} / \mathrm{L})$, 
creatinine $(\mathrm{mg} / \mathrm{dL})$, total bilirubin $(\mathrm{mg} / \mathrm{dL})$, aspartate aminotransferase (IU/L), alanine aminotransferase (IU/L), and Kidney Donor Profile Index (\%).

The number and types of organ(s) procured from each donor were reviewed. Data from 35 recipients of the 36 organs procured from the 10 facial VCA ( 2 of which were multi-VCA) donors during the study period were analyzed. Specifically, patient status and cause of death, graft status and cause of graft failure, surrogate markers of graft function, and organ-specific laboratory values reported at follow-up were analyzed. We defined delayed graft function (DGF) as per SRTR definition, specifically, the initiation of dialysis within the first week posttransplant. All statistical analyses were performed using Excel 2007 (Microsoft Corporation, Redmond, WA).

\section{Donor Operation}

The New England Organ Bank has collaborated in all of our center's efforts to recover and transplant VCA. Similarly, Lifebanc of Ohio and The Living Legacy Foundation of Maryland have been involved and instrumental to their center's success. In our center, preoperative conference calls and extensive discussions are conducted between the New England Organ Bank, our center, and solid organ procurement teams before all procedures. Discussion and planning include topics, such as the timing and sequence of procedure(s), allocation of key accessory vessels, and the need for an onsite, standby abdominal transplant surgeon until the arrival of the liver recovery team.

Although our centers (Boston and Cleveland) have previously described our standard protocol for donor allograft procurement, ${ }^{4}$ our experiences have not been uniform. Each procedure has been guided by the unique needs of each VCA recipient and by the needs of the solid organ recovery team. ${ }^{11,12}$ Briefly, as heart-beating donors are typically he modynamically stable, procurement of the facial allograft starts first, with the safe procurement of lifesaving organs taking priority over recovery of nonlifesaving VCAs (eg, face and upper extremities). In addition, free fasciocutaneous radial forearm flaps were recovered from donors 1 to 3,5 , and 6 and transplanted into the facial VCA recipients at secondary sites and used for remote-site immune monitoring and functional purposes. ${ }^{13}$ University of Wisconsin solution was used as the cold preservative solution for 8 of the 9 reported VCAs. The first Cleveland case did not perfuse the allograft with preservation solution. Cleveland's donor procurement team consisted of a team of 4 surgeons with expertise in microsurgery, head and neck, and craniofacial surgery. The facial VCA procurement was followed by the recovery of solid organs. ${ }^{5}$ Similarly, our facial VCA donor recovery teams ranged from 4 to 5 surgeons, with a primary surgeon on each side of the face and 1 to 2 assistants. Upper extremity donor recovery teams include 2 surgeons per extremity. Maryland's donor procurement team consisted of 2 surgeons with 2 assistants. Their donor procurement protocol calls for a "face-first, concurrent completion" approach with preemptive thoracic and abdominal dissection that prioritizes the safety of solid organs and allows for a speedy procurement in the event of sudden donor instability. Their proposed algorithm has been detailed in a previous publication. ${ }^{9}$ 
Detailed intraoperative data, such as donor surgery location, length of facial allograft procurement, components of the facial allograft, mean intraoperative hemodynamic parameters, vasopressor requirements, and blood products transfused, were also reviewed.

\section{RESULTS}

A total of 10 brain-dead donors ( 7 men and 3 women), with mean age of 39.7 years (range, 21-60 years) and average Kidney Donor Profile Index 45.7\% (range, 3-96\%) underwent facial VCA procurement with donors 4 and 7 also undergoing concomitant bilateral upper extremity procurement. All donors also underwent procurement of 1 or more solid organs. The details of donor characteristics and operative data are displayed in Tables 1 and 2, respectively. A total of 36 solid organs were recovered and transplanted into 35 recipients, with 1 recipient being a simultaneous kidney-pancreas $(K P)$ : heart $(n=4)$, lung $(n=3)$, kidney ( $\mathrm{n}=16$ [15 single and 1 as a KP]), liver ( $\mathrm{n}=10 ; 8$ full, 2 split), pancreas ( $\mathrm{n}=2[1$ single and 1 as a KP]), intestine $(n=1)$.

\section{Donor Operation}

All VCA procurements were commenced before the recovery of lifesaving organs. In 1 case (donor 2), we performed aportion of the recovery after circulatory death. Six of 10 donor surgeries were performed at outside institutions, all on brain-dead donors. Mean operative duration for facial VCA recovery was 6.9 hours (range, 4-13.25 hours). Low-dose vasopressors were required in 7 of 10 donors with over-all stable hemodynamic parameters. Intraoperative blood transfusions were required for 5 donors. Donor 1 received a total of 6 units of packed red blood cells (PRBC), donor 4 received 1 unit of PRBC, and donor 7 received 2 units of $\mathrm{PRBC}$ in the operating room before any recovery for he matocrit of $18 \%$ and 1 additional unit during solid organ recovery for hematocrit of 20\%. Donor 10, a partial osteomyocutaneous facial allograft donor, received 7 units of PRBC, 4 units of plasma and 3 units of platelets with the need for vasopressors and at 1 point also needing Nitroglycerin. Time from facial VCA removal to aortic cross-clamping was 1 hour and 45 minutes. Lastly, 1 full-face osteomyocutaneous donor required 5 units of PRBC in addition to 2 units of plasma without the need for vasopressors (see Table 2). The details of all structures included in each facial allograft are summarized in Table 2.

\section{Other Nonfacial VCA Recovery}

Bilateral upper extremities were procured in donors 4 and 7 . This took place concomitantly with facial VCA procurement and before solid organ recovery. Total procurement times were 8.5 hours for both cases of combined face and bilateral upper extremities. Details of donor 4 's procurement have been previously published. ${ }^{14}$ For donor 7 , the right upper extremity was procured below the elbow level, whereas the left was performed just above the elbow level in similar fashion with minimal blood loss under tourniquet control. Sentinel flaps in the remaining 5 donors (donors 1 to 3,5 , and 6) were recovered as free fasciocutaneous radial forearm flaps. 
Kidney

Recipient survival rates were $100 \%$, and graft survival rates were $94 \%$ for kidneys ( $\mathrm{n}=16$ ) at a median follow-up time of 33 months (range, 6-72 months). Acute rejection accounted for the only case of graft failure by 1 month. Mean serum creatinine was $1.51 \mathrm{mg} / \mathrm{dL}$ at median follow-up. Delayed graft function developed in 7 of 16 (44\%) kidneys with a median cold ischemia time of 11.6 hours (14 of 16 kidneys reported cold ischemia times; range, 6.639 hours). Median cold ischemia time in DGF cases was 21.5 hours (range, 9-39 hours). Median cold ischemia time in non-DGF cases was 11.2 hours (range, 6.6-26 hours). Median length of stay for all kidney recipients was 9 days (range, 3-30 days). There were 2 kidneys, 1 from each donor (donors 4 and 10) that were recovered with the intent to transplant, but were not transplanted due to poor pump parameters.

\section{Liver}

Recipient and graft survival rates were $90 \%(\mathrm{n}=10)$ at a median follow-up time of 27.5 months (range, 6-72 months). All were functioning with a mean total bilirubin $0.6 \mathrm{mg} / \mathrm{dL}$. Liver grafts had a median cold ischemia time of 4.7 hours (range, 2.6-16 hours) and median length of stay of 12.5 days (range, 10-26 days). One liver recipient died at 22 months from unknown causes. The recipient was evaluated at 19 months posttransplant, with a serum total bilirubin level of $0.4 \mathrm{mg} / \mathrm{dL}$.

\section{Pancreas and Intestine}

There was 1 pancreatic graft recovered and successfully transplanted into 1 recipient. The patient was discharged from the hospital after 8 days and remained well at their 6 month follow-up with no transplant-related infectious or surgical complications (eg, pancreatic abscess, anastomotic leak, pancreatitis). No specific data regarding insulin requirements were provided. The intestine recipient was on oral feeds without the need for parenteral nutrition or tube feeds at 6-month follow-up.

\section{Kidney-Pancreas}

There was 1 simultaneous kidney-pancreas transplant. The recipient and kidney allograft are alive at 33 months follow-up with a serum creatinine of $1.0 \mathrm{mg} / \mathrm{dL}$. The cold is chemia time was 16.9 hours, and there was no delayed graft function. Unfortunately, the recipient suffered a pancreatic bleed that led to graft failure due to graft/vascular thrombosis on postoperative day 3 and had to resume insulin. The length of stay was 16 days.

\section{Heart and Lung}

Recipient and graft survival rates for hearts were $75 \%(\mathrm{n}=4)$ with an average follow-up time of 14.75 months (range, 6-36 months). One heart recipient is alive at 36 months follow-up with an ejection fraction of $65 \%$ without the need for subsequent renal transplant or cardiac pacing. One heart recipient had stable ejection fraction of $55 \%$, but passed away from leukemia 10 months posttransplant. There is missing ejection fraction data for 2 of 4 heart recipients, though follow-up at 6 and 7 months confirm both the recipient and allograft are alive. 
Recipient and graft survival rates for lungs were $100 \%(\mathrm{n}=3)$ with an average follow-up time of 16 months (range, 6-35 months). All lungs were transplanted as single lungs. One single lung recipient is alive at 35 months follow-up with forced expiratory volume in 1 second of $83 \%$ and without the need for supplemental oxygen. There is missing forced expiratory volume in 1 second data for the other 2 recipients, though follow-up at 6 and 7 months confirm both the recipient and allograft are alive.

\section{DISCUSSION}

In this study, we demonstrate that despite additional surgical time, logistical considerations, and the potential for blood product transfusion, facial and simultaneous multi-VCA procurement do not appear to adversely affect the short-term or long-term outcomes of transplant recipients and solid organs recovered from the same donor. Notably, our cohort DGF rate of 44\% (7 of 16 kidneys) was observed with a median cold ischemia time of 21.5 hours (range, 9-39 hours) versus a median cold ischemia time of 11.2 hours (range, 6.6-26.0 hours) in non-DGF kidneys. These results are consistent with a common observation in the kidney transplantation literature, where prolonged cold ischemia time is related to increased rates of DGF. However, recent literature suggests that cold ischemia time-induced DGF does not impact long-term outcomes of kidney allografts, even in the setting of donor acute kidney injury. ${ }^{15,16}$ More importantly, VCA procurement should not affect these ischemia times unless of course, it was to directly (or indirectly) cause sudden hemodynamic instability and circulatory death which was not observed in our cohort of patients.

In regards to our donor surgeries, we have observed a gradual improvement in the time of dissection and overall stable procedure times with increasing experience, despite the need for more complex maxillary and mandibular procurements. Additionally, intraoperative requirement of multiple blood transfusions has been significant only in our first case where a total of 6 units PRBC were administered, despite temporarily clamping bilateral external carotid arteries before osteotomies. This donor was a special case due to the requirement of bivalirudin, a direct thrombin inhibitor, for a history of heparin-induced thrombocytopenia. ${ }^{17}$ Our procedure times have been commensurate with the extent of the facial allograft procurement based on the recipients' needs. For example, recovery of maxilla was necessary in donors 1 and 4 and additional mandible in donors 6 and 7. Likewise, this is also seen with Cleveland and Maryland experiences because these were not only the center's first cases but also involved an extensive osteomyocutaneous facial VCA procurement.

In regards to concomitant recovery of other composite tissues, there were 2 cases (donors 4 and 7) that included the procurement of bilateral upper extremities. Bilateral upper extremity procurement is shorter in duration, takes place under tourniquet with minimal blood loss, and introduces less complex coordination and planning when compared with a facial VCA procurement. ${ }^{18}$ Blood loss during the period of tourniquet release was minimal in both donors 4 and $7 .{ }^{14}$ The procurement of bilateral upper extremities in donors 4 and 7 brought the total VCA procurement times preceding solid organ recovery to 8.5 hours, approximately 1.5 hours more than the average facial VCA procurement.

Transplantation. Author manuscript; available in PMC 2019 January 25. 
Our second donor procurement was the first time that we recovered the whole facial allograft. In this case, pressing needs for solid organ recovery did not allow adequate time for complete facial allograft procurement before aortic cross-clamping. Therefore, expeditious neck dissection and identification of our vascular pedicle was achieved before aortic cross-clamping and subsequent recovery of lifesaving organs. This was our only case where the facial allograft was partially recovered after circulatory death. Though our center works to maintain the cold ischemia of VCAs to less than 4 hours, we acknowledge that lifesaving organs are the priority under these circumstances. The intimate working relationship with our regional organ procurement organization has been instrumental to our success. Working collaboratively with the New England Organ Bank, we have been able to safely plan our donor operations before the recovery of lifesaving organs. The 2 multi-VCA and multi-solid organ procurement emphasize the importance of preoperative discussion and planning guided by the organ procurement organization.

There are several limitations to our study. One challenge of studying the potential impact of VCA procurement on the outcomes of solid organs is the multifactorial nature of long-term allograft function and survival. Characteristics such as age, race, medical history, donation after circulatory death, history of hepatitis $\mathrm{C}$, cold ischemia time, organ preservation techniques (eg, hypothermic machine perfusion versus static cold storage) are among the many factors that can impact allograft outcomes. ${ }^{19,20}$ Our understanding of donor characteristics continues to evolve and has led to the development of novel scoring systems and risk models for organs such as kidneys with the Kidney Donor Profile Index ${ }^{21,22}$ and liver with the Eurotransplant Donor Risk Index. ${ }^{23}$ Our limited sample size of 10 donors, 36 solid organs, and un-known condition of the recipients cannot account for all known and unknown predictors of posttransplantation patient survival and graft function. In addition, the retrospective nature of our investigation is subject to the limitations entailed by such a study design.

Although our study is limited and only includes 10 of the 37 face transplants worldwide, to our knowledge, this is the first study to not only review national VCA donor surgery experiences but also the potential impact of facial and multi-VCA procurement on the outcomes of solid organ recipients and allografts in the English language literature. An important step for the field of VCA came in July 2013 when the Department of Health and Human Services issued its Final Rule to redefine VCA as organs rather than tissues. ${ }^{24}$ Now under the oversight of Organ Procurement and Transplantation Network, there have been numerous national policies and standards regarding the practices of VCA procurement and transplantation. In stark contrast to solid organ transplantation, there is no systematic, centralized data collection repository for VCA transplants but work is ongoing for the standardization and reporting of outcomes. We hope that this experience can add to national standards being developed for face and other VCA transplants.

\section{CONCLUSIONS}

The advent and continued progress of vascularized composite allotransplantation has ushered in a new clinical arena in the field of transplantation. One major aspect that remains critical to its progress is the planning and safe execution of the donor procurement. Here we

Transplantation. Author manuscript; available in PMC 2019 January 25. 
show that the procurement of facial VCA and simultaneous upper extremities is safe and does not appear to interfere with the procurement and outcomes of lifesaving, solid organ transplants. As always, excellent coordination and communication between the organ recovery teams remains paramount with guidance and support from the respective organ procurement organization.

\section{ACKNOWLEDGMENTS}

The authors would like to thank the donors, their families, the New England Organ Bank, Lifebanc of Ohio, The Living Legacy Foundation of Maryland, the respective solid organ procurement teams and hospitals, and the entire Brigham and Women's Face and Upper Extremity Transplant team.

The interpretation and reporting of these data are the responsibility of the authors, and the contents do not necessarily represent the official policy of or interpretation by the Scientific Registry of Transplant Recipients, the U.S. government, or the Health Resources and Services Administration (HRSA).

Funding was provided by the U.S. Department of Defense (to B.P., E.M.B., N.K., S.G.T., M.J.C., and S.G.T. Partial support was received from the Department of Defense Biomedical Translational Initiative research contract W911QY-09-C-0216) and the National Institutes of Health (to S.G.T., RO1AG039449).

\section{REFERENCES}

1. Khalifian S, Brazio PS, Mohan R, et al. Facial transplantation: the first 9 years. Lancet. 2014;384:2153-2163. [PubMed: 24783986]

2. Pomahac B, Pribaz J, Eriksson E, et al. Three patients with full facial transplantation. N Engl J Med. 2012;366:715-722. [PubMed: 22204672]

3. Lantieri L, Hivelin M, Audard V, et al. Feasibility, reproducibility, risks and benefits of face transplantation: a prospective study of outcomes. Am J Transplant. 2011;11:367-378. [PubMed: 21272240]

4. Pomahac B, Papay F, Bueno EM, et al. Donor facial composite allograft recovery operation: Cleveland and Boston experiences. Plast Reconstr Surg. 2012;129:461e-467e.

5. Siemionow M, Ozturk C. Donor operation for face transplantation. J Reconstr Microsurg. 2012;28:35-42. [PubMed: 21374550]

6. Meningaud JP, Paraskevas A, Ingallina F, et al. Face transplant graft procurement: a preclinical and clinical study. Plast Reconstr Surg. 2008;122:1383-1389. [PubMed: 18971721]

7. Alam DS, Papay F, Djohan R, et al. The technical and anatomical aspects of the World's first neartotal human face and maxilla transplant. Arch Facial Plast Surg. 2009;11:369-377. [PubMed: 19917898]

8. Gomez-Cia T, Infante-Cossio P, Sicilia-Castro D, et al. Sequence of multiorgan procurement involving face allograft. Am J Transplant. 2011;11:2261. [PubMed: 21812926]

9. Brazio PS, Barth RN, Bojovic B, et al. Algorithm for total face and multiorgan procurement from a brain-dead donor. Am J Transplant. 2013;13:2743-2749. [PubMed: 23915309]

10. Bueno J, Barret JP, Serracanta J, et al. Logistics and strategy of multiorgan procurement involving total face allograft. Am J Transplant. 2011;11:1091-1097. [PubMed: 21443675]

11. Pomahac B, Pribaz JJ, Bueno EM, et al. Novel surgical technique for full face transplantation. Plast Reconstr Surg. 2012;130:549-555. [PubMed: 22575856]

12. Singhal D, Pribaz JJ, Pomahac B. The Brigham and Women's Hospital face transplant program: a look back. Plast Reconstr Surg. 2012;129:81e-8e.

13. Kueckelhaus M, Fischer S, Lian CG, et al. Utility of sentinel flaps in assessing facial allograft rejection. Plast Reconstr Surg. 2015;135: 250-258. [PubMed: 25255116]

14. Carty MJ, Hivelin M, Dumontier C, et al. Lessons learned from simultaneous face and bilateral hand allotransplantation. Plast Reconstr Surg. 2013;132:423-432. [PubMed: 23584623]

15. Kayler LK, Srinivas TR, Schold JD. Influence of CIT-induced DGF on kidney transplant outcomes. Am J Transplant. 2011;11:2657-2664. [PubMed: 22051325] 
16. Xia Y, Friedmann P, Cortes CM, et al. Influence of cold ischemia time in combination with donor acute kidney injury on kidney transplantation outcomes. J Am Coll Surg. 2015;221:532-538. [PubMed: 26206650]

17. Edrich T, Pomahac B, Lu JT, et al. Perioperative management of partial face transplantation involving a heparin antibody-positive donor. J Clin Anesth. 2011;23:318-321. [PubMed: 21663819]

18. Banegas RN, Moreno R, Duggal A, et al. Surgical aspects of donor hand recovery for transplantation. J Reconstr Microsurg. 2012;28:21-26. [PubMed: 21861253]

19. Legendre C, Canaud G, Martinez F Factors influencing long-term outcome after kidney transplantation. Transpl Int. 2014;27:19-27. [PubMed: 24138291]

20. Dubbeld J, van Hoek B, Ringers J, et al. Biliary complications after liver transplantation from donation after cardiac death donors: an analysis of risk factors and long-term outcome from a single center. Ann Surg.2015;261:e64. [PubMed: 24401915]

21. Rao PS, Schaubel DE, Guidinger MK, et al. A comprehensive risk quantification score for deceased donor kidneys: the kidney donor risk index.Transplantation.2009;88:231-236. [PubMed: 19623019]

22. Gupta A, Chen G, Kaplan B. KDPI and donor selection. Am J Transplant.2014;14:2444-2445. [PubMed: 25155434]

23. Braat AE, Blok JJ, Putter H, et al. The Eurotransplant donor risk index in liver transplantation: ETDRI. Am J Transplant. 2012;12:2789-2796. [PubMed: 22823098]

24. Health Resources and Services Administration (HRSA), Department of Health and Human Services (HHS). Organ procurement and transplantation network. Final rule. Fed Regist. 2013;78:40033-40042. [PubMed: 23833809] 


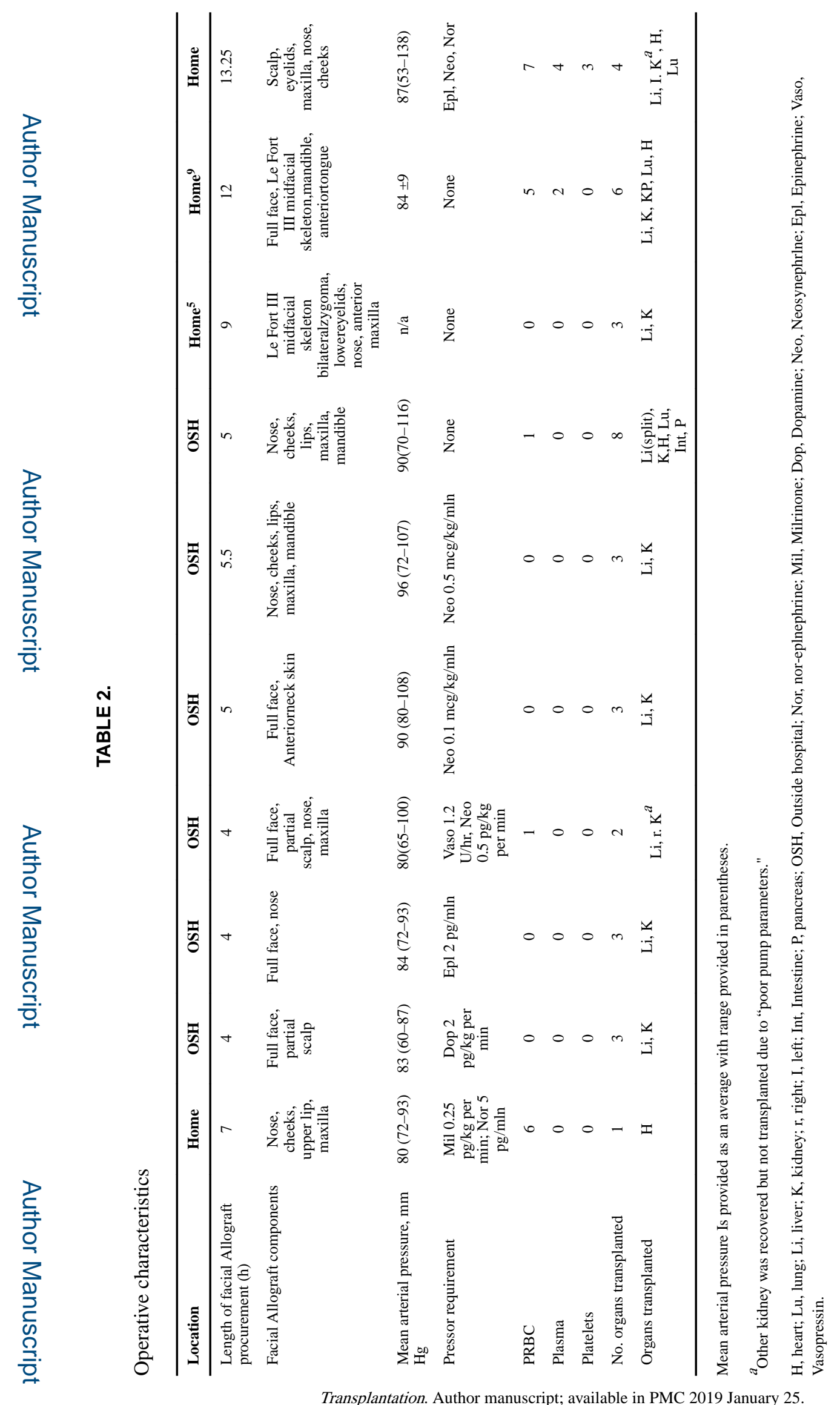

